CLINICAL STUDY

\title{
Negative illness perceptions are associated with impaired quality of life in patients after long-term remission of Cushing's syndrome
}

\author{
Jitske Tiemensma ${ }^{1}$, Adrian A Kaptein ${ }^{2}$, Alberto M Pereira ${ }^{1}$, Johannes W A Smit ${ }^{1}$, Johannes A Romijn ${ }^{1}$ \\ and Nienke R Biermasz ${ }^{1}$ \\ Departments of ${ }^{1}$ Endocrinology and Metabolism and ${ }^{2}$ Medical Psychology, Leiden University Medical Center, Albinusdreef 2, 2333 ZA Leiden, \\ The Netherlands \\ (Correspondence should be addressed to J Tiemensma; Email: j.tiemensma@lumc.nl)
}

\begin{abstract}
Objective: Illness perceptions pertain to the pattern of beliefs patients develop about their illness. Illness perceptions are determinants of quality of life $(\mathrm{QoL})$. Factors contributing to persisting impaired QoL after Cushing's syndrome (CS) remain largely unknown. Therefore, the objective of this study was to explore illness perceptions, as potentially modifiable psychological factors, in relation to QoL in patients with long-term remission of CS.

Design: Cross-sectional study.

Methods: We included patients with long-term remission of CS $(n=52)$. Illness perceptions were evaluated using the Illness Perception Questionnaire (IPQ)-Revised, and QoL was measured using the physical symptom checklist, EuroQoL-5D (EQ-5D), and the CushingQoL. Reference data were derived from recent studies and included patients with vestibular schwannoma $(n=80)$, acute $(n=35)$ or chronic $(n=63)$ pain, and chronic obstructive pulmonary disease (COPD; $n=171)$.

Results: Illness perceptions showed a strong correlation with QoL. Patients with CS scored distinctively more negative on the IPQ compared with patients with vestibular schwannoma and patients with acute pain, and also reported more illness-related complaints (all $P<0.01$ ). There were also some differences in illness perceptions between patients with CS and patients with chronic pain and patients with COPD, but there was no distinct pattern.

Conclusions: Patients after long-term remission of CS report more negative illness perceptions compared with patients with other acute or chronic conditions. Further research is needed to assess whether QoL in CS can be improved by addressing these illness perceptions, for example, by a self-management intervention program.
\end{abstract}

European Journal of Endocrinology 165 527-535

\section{Introduction}

Cushing's syndrome (CS) is characterized by excessive glucocorticoid levels, mostly caused by ACTH-producing pituitary adenomas, but in one-third of the cases by ectopic ACTH-producing neuroendocrine tumors or by adrenal adenomas/carcinomas. Following successful treatment of hypercortisolism, signs and symptoms improve substantially. However, prolonged, excessive exposure to glucocorticoids may have long-lasting adverse effects on behavioral and cognitive functions, due to functional and structural alterations in specific brain target areas (1-4). Furthermore, these patients do not completely return to their premorbid level of functioning, and quality of life (QoL) is persistently impaired despite long-term cure of CS (5). Although the decreased QoL may originate from persisting limitations due to irreversible effects of excessive glucocorticoid exposure, an alternative hypothesis is that the psychological impact of suffering from this disease results in QoL reduction. We recently reported that coping strategies are indeed ineffective in patients with pituitary disease (6). Persistent inappropriate thoughts about the disease and/or its treatment can influence general well-being. Patients and their doctors may have (totally) discrepant perceptions of the severity of the disease and the success of treatment. This concept has not been elaborated for endocrine diseases and can be studied by measuring illness perceptions.

Illness perceptions pertain to the way patients make sense of, and respond to, their illness. Illness perceptions are conceptualized in the common-sense model (CSM) of self-regulation. The CSM explains how patients generate both cognitive representations of, and emotional reactions to, their illness, integrating internal and external stimulus information with their 
pre-existing illness-theory (7). Leventhal et al. (8) designed the CSM which starts from the premise that individuals are active problem solvers, who make sense of a threat to their health by developing a cognitive representation of the threat, which determines how the individual responds. Patients cluster these representations or ideas about the illness around five cognitive components: i) identity: the label that is used by the individual to describe the condition and the associated symptoms; ii) cause: personal ideas about the cause of the condition; iii) timeline: expectations about the likely duration of the condition; iv) consequences: the physical, psychological, and social effects of the condition; and v) cure/control: the extent to which the condition is amenable to cure and/or control. These components form the illness representations, which determine the patient's coping procedures (9). The source of patient's perceptions is diverse. It can be based on information from doctors, relatives, friends, or media, but also from first-hand experiences in close proximity to someone who suffers from an illness. Therefore, patients' illness perceptions are subjective, may be partly or completely incorrect, and do not necessarily represent the medical status of the disease.

At present, there are no studies that have evaluated illness perceptions in patients with long-term remission of CS. Therefore, the aim of this study is to explore the illness perceptions of patients after long-term remission of CS in relation to reported QoL using the Illness Perception Questionnaire-Revised (IPQ-R) and several QoL questionnaires. The IPQ-R questionnaire assesses perceptions on each of the five components by asking the patient for their own beliefs about the CS. Since there are no previous studies in endocrine patients we compared findings to several Dutch reference groups.

\section{Patients and methods}

\section{Patients}

CS had been diagnosed based on internationally agreed guidelines, i.e. the clinical manifestations and positive biochemical tests including increased urinary excretion rates of free cortisol, decreased overnight suppression by dexamethasone $(1 \mathrm{mg})$ and, since 2004 , elevated midnight salivary cortisol values. All patients had been treated by transsphenoidal surgery or adrenalectomy, if necessary followed by repeated surgery and/or postoperative radiotherapy. Cure of CS was defined by normal overnight suppression of plasma cortisol levels $(<50 \mathrm{nmol} / \mathrm{l})$ after administration of dexamethasone $(1 \mathrm{mg})$ and normal $24 \mathrm{~h}$ urinary excretion rates of cortisol $(<220 \mathrm{nmol} / 24 \mathrm{~h})$. Hydrocortisone independency was defined as a normal cortisol response to CRH or insulin-tolerance test (ITT).

All patients were followed at our outpatient department. Patients were monitored for (recurrence of) disease, according to appropriate dynamic tests. Pituitary function was monitored and pituitary hormone replacement was prescribed dependent on the results of the yearly evaluation of pituitary functions. In case of corticotrope insufficiency, confirmed by ITT or $\mathrm{CRH}$ test, the average dose of hydrocortisone was $20 \mathrm{mg} /$ day divided into two to three dosages. Evaluation of GH deficiency was performed by ITT and/or GHRH-arginine test, only in patients under the age of 70 years and only after at least 2 years of remission. Somatotrope insufficiency was treated with rhGH replacement, aiming at IGF1 concentrations in the normal range for age. In addition, free thyroxine $\left(\mathrm{T}_{4}\right)$ and testosterone levels (in male patients) were assessed. If results were below the lower limit of the respective reference ranges, substitution with $\mathrm{L}_{-} \mathrm{T}_{4}$ and/or testosterone was prescribed. In the case of amenorrhea and low estradiol levels in premenopausal women, estrogen replacement was provided. Progesterone replacement was also provided to women with an intact uterus.

Education level was based on the Dutch education system, which is comparable to the International Standard Classification of Education. Low education level was defined as primary education to lower secondary education. Medium education level incorporated (upper) secondary education to post-secondary non-tertiary education, while high level education was defined as the first stage of tertiary education to the second stage of tertiary education.

Inclusion criteria for this study were age $>18$ years and remission defined by strict biochemical criteria for at least 1 year. The protocol was approved by the LUMC medical ethics committee.

\section{Methods}

Protocol We performed a clinical chart review of 77 patients with CS. All were in remission at the time of this study for at least 1 year. The long-term treatment outcome of these patients has been characterized and described in detail (10). We invited these patients to participate in this study. Twenty-five patients $(32 \%)$ refused to participate and 52 patients $(68 \%)$ participated in this study and completed all questionnaires. The clinical characteristics of the subjects, who did not participate, did not differ from those of the participants. Patients were asked to complete questionnaires on illness perceptions and QoL at home and return these questionnaires in a prepaid envelope.

Illness Perception Questionnaire-Revised The IPQ-R was used to measure cognitive and emotional representations of CS $(11,12)$. The questionnaire was developed to assess the components of the illness representation of Leventhal's Self-Regulatory Model and is frequently used to study illness perceptions in 
chronic conditions (13-17). The IPQ-R is divided into three sections. The first part consists of the illness identity dimension, with a list of 14 general commonly occurring symptoms and 13 symptoms commonly occurring in CS. Patients were asked to rate whether or not they experienced the symptoms, and if they believe the symptom to be related to CS (yes/no). The summed up yes-rated items of the disease-related symptoms were used in the analysis.

The second part of the questionnaire, assessing illness perception dimensions, consists of 38 statements concerning views on the illness, scored on a five-point Likert scale (from strongly disagree to strongly agree). The questions were transformed to seven dimensions: timeline acute/chronic (beliefs about the chronic nature of the condition), timeline cyclical (beliefs regarding the cyclical nature of the condition), consequences (negative consequences of the disease), emotional representations (the likelihood to seek medical care), personal control (perceived personal controllability of the disease), treatment control (perceived treatment controllability of the disease), and illness coherence (personal understanding of the disease). A higher score indicates a stronger belief in that particular dimension. The third and final part of the questionnaire was about the causal attributions. This section consisted of 18 statements concerning possible causes that patients considered to be contributing to their disease, scored on a five-point Likert scale (strongly disagree to strongly agree).

As recommended by the developers of the questionnaire, a principal component analysis with varimax rotation was performed on the causal items to cluster variables with shared variance (11). The analysis produced four factors accounting for $72 \%$ of the variance. The first factor labeled "psychological attributions' accounted for $31 \%$ of the variance and consisted of the items emotional state, personality, overwork, stress or worries, mental attitude, family problems/worries, ageing, altered immunity, and own behavior. The second factor labeled 'risk factors' accounted for $22 \%$ of the variance and consisted of the items pollution in environment, diet or eating habits, bacteria or virus, accident/injury, and poor medical care. The third factor 'behavioral attributions' accounted for $11 \%$ of the variance and consisted of the items smoking and alcohol use. The fourth factor 'chance' accounted for $9 \%$ of the variance and consisted of the items hereditary and chance/bad luck. This fourth factor demonstrated insufficient internal reliability $(\alpha<0.50)$, and was excluded from further analysis. Higher scores on the other three causal subscales indicate stronger beliefs in those attributions in causing CS.

QoL questionnaires Physical symptom checklist. This is a checklist of 55 physical symptoms that are mentioned in the DSM-III classification (18). The symptoms cover most organ systems. There are 11 general/neurological items, ten autonomic items, eight musculoskeletal/pain items, 13 gastrointestinal items, five genital items, and four items about feeling hot/cold. The presence of symptoms is rated on a severity scale from 0 to 3 . We excluded the gender-specific items $(n=4)$ from the analyses to rule out bias by gender. The total symptom score ranges from 0 to 153. A higher score indicates more (severe) physical symptoms in the preceding week (19).

EuroQoL-5D. This QoL questionnaire measures five health dimensions: mobility, self-care, usual activities, pain/discomfort, and anxiety/depression. Scores are expressed on a 1-3 scale per dimension, with a higher score indicating a worse QoL. The questionnaire also includes a visual analog scale (VAS) that comprises a standard vertical $20 \mathrm{~cm}$ scale (similar to a thermometer) for recording an individual's rating for their current health-related QoL state (20). The VAS score ranges from 0 to 100 , with higher scores indicating a better QoL.

CushingQoL. This is a disease-specific QoL questionnaire specifically designed to assess QoL in patients with CS. The questionnaire consists of 12 questions on a fivepoint Likert scale ranging from always to never. The total score ranges from 12 to 60 , with a lower score indicating a greater impact on health-related QoL (21).

Three patients did not complete all QoL questionnaires.

Reference populations Since the general population is not assumed to have an illness, there are no normative values of the IPQ-R available for the general population. Scores of patients with acute and chronic pain may be used instead (22). The other reference groups were chosen based on available data of the IPQ-R in Dutch samples.

Patients with acute and chronic pain are described in the paper that presents the revised version of the IPQ (11). The reference group with acute pain consisted of 35 subjects who were recruited from a private practice for physical therapy (20 men, 15 women, mean age of $36 \pm 12$ years). The patients presented with a first time peripheral painful injury that had been present for $<6$ weeks. The reference group of patients with chronic pain consisted of 63 subjects $(26$ men and 37 women, with a mean age of $54 \pm 11$ years) who were recruited from hospital based chronic pain clinics. All patients experienced pain for longer than 3 months that was unexplained by medical signs alone.

The third reference group consisted of 171 Dutch patients (112 men and 59 women, mean age $66 \pm 10$ years) suffering from chronic obstructive pulmonary disease (COPD) (23). A chest physician diagnosed all patients as suffering from emphysema and/or chronic bronchitis.

The fourth reference group consisted of 80 patients with vestibular schwannoma who just had been told of the diagnosis, but who had not received a treatment 
Table 1 Clinical characteristics. Data are presented as mean \pm s.D. or number (\%).

\begin{tabular}{|c|c|c|c|}
\hline & $\begin{array}{l}\text { Total } \\
\text { patients } \\
\text { with CS } \\
(n=52)\end{array}$ & $\begin{array}{l}\text { HC } \\
\text { dependent } \\
(n=30)\end{array}$ & $\begin{array}{l}\text { HC not } \\
\text { dependent } \\
(n=22)\end{array}$ \\
\hline Gender (male/female) & $7 / 45$ & $5 / 25$ & $2 / 20$ \\
\hline \\
\hline Low & $16(31 \%)$ & $9(30 \%)$ & $7(32 \%)$ \\
\hline Medium & $18(35 \%)$ & $11(37 \%)$ & $7(32 \%)$ \\
\hline High & $16(31 \%)$ & $9(30 \%)$ & $7(32 \%)$ \\
\hline Unknown & $2(4 \%)$ & $1(3 \%)$ & $1(5 \%)$ \\
\hline \multicolumn{4}{|l|}{ Surgery, $n(\%)$} \\
\hline Transsphenoidal & $41(79 \%)$ & $16(53 \%)$ & 18 (82\%) \\
\hline Adrenal & $11(21 \%)$ & $7(23 \%)$ & $4(18 \%)$ \\
\hline Both & $7(14 \%)$ & $7(23 \%)$ & $0(0 \%)$ \\
\hline PO radiotherapy, $n(\%)$ & $14(27 \%)$ & $10(33 \%)$ & $4(18 \%)$ \\
\hline Dur. of remission (years) & $16 \pm 12$ & $18 \pm 13$ & $14 \pm 10$ \\
\hline Dur. of follow-up (years) & $17 \pm 12$ & $18 \pm 13$ & $15 \pm 10$ \\
\hline \multicolumn{4}{|l|}{ Hypopituitarism, n (\%) } \\
\hline Any axis & $32(62 \%)$ & $30(100 \%)$ & $2(9 \%)$ \\
\hline $\mathrm{GH}$ & $23(44 \%)$ & $21(70 \%)$ & $1(5 \%)$ \\
\hline LH/FSH & $14(27 \%)$ & $13(43 \%)$ & $2(9 \%)$ \\
\hline TSH & 19 (37\%) & $17(57 \%)$ & $2(9 \%)$ \\
\hline $\mathrm{ADH}$ & $8(15 \%)$ & $8(27 \%)$ & $0(0 \%)$ \\
\hline HC substitution, $n(\%)$ & $30(58 \%)$ & $30(100 \%)$ & $0(0 \%)$ \\
\hline
\end{tabular}

$\mathrm{HC}$, hydrocortisone; PO, postoperative; Dur., duration.

proposal at the time of the study (36 men, 43 women, and one anonymous responder. Mean age 57 years, range 26-79 years) (22). Thirty-eight percent of the patients suffered from an intracanalicular tumor, and $8 \%$ from a cystic component. The symptoms experienced by these patients included tinnitus, unsteadiness, vertigo, headache, and earache.

\section{Statistical analysis}

Data were analyzed using PASW Statistics version 17.0.2 (SPSS, Inc., Chicago, IL, USA). All data were presented as mean \pm s.D., unless mentioned otherwise. The primary analysis comprised the relationship between illness perceptions and QoL. Partial correlations were calculated controlling for duration of remission. The level of significance for this analysis was set at $P \leq 0.05$. The comparison of patients with a relatively better QoL and patients with a worse QoL consisted of a Student's t-test with the level of significance set at $P \leq 0.01$, because of multiple comparisons. The possible effects of duration of remission and duration of follow-up were explored by linear regression analysis. The standardized $\beta$ coefficients of this analysis were reported.

Secondary analysis comprised the comparison of results in patients with long-term cure of CS and of results in various reference groups. Means were calculated for all subscales of the IPQ and compared between groups by Student's t-test. Patients with cure of CS with and without hydrocortisone substitution were also compared by a Student's t-test, while the different treatment modalities were compared using an ANOVA with a post hoc analysis when appropriate. Since multiple comparisons were used, the level of significance was set at $P \leq 0.01$.

\section{Results}

\section{Sociodemographic and clinical characteristics}

Clinical characteristics of the patients are detailed in Table 1. Forty-six patients (88\%) had pituitary ACTHdependent hypercortisolism and six patients $(12 \%)$ had CS due to adrenal tumor. There were no significant differences between patients who had been treated for CS caused by an adrenal cortisol-producing tumor and patients who had been treated for Cushing's disease neither on the IPQ nor on any of the QoL questionnaires (Fig. 1).

Thirty-four patients $(65 \%)$ had been treated by transsphenoidal surgery, seven patients $(14 \%)$ by both transsphenoidal surgery and adrenalectomy, and 11 patients $(21 \%)$ had been treated by adrenalectomy. Fourteen patients $(27 \%)$ had been treated by additional pituitary irradiation for persistent disease after surgery. At the time of this study, all patients were in remission and the mean duration of remission was $16 \pm 12$ years (range 2-46 years). Twenty-three patients were $\mathrm{GH}$ deficient, of whom 21 received GH therapy. A total of 32 patients $(62 \%)$ were treated for some degree of pituitary insufficiency and 30 patients $(58 \%)$ were substituted with hydrocortisone. Figure 1 shows the self-reported symptoms of various organ systems in patients after long-term remission of CS. Most complaints involved musculoskeletal pain (Fig. 1).

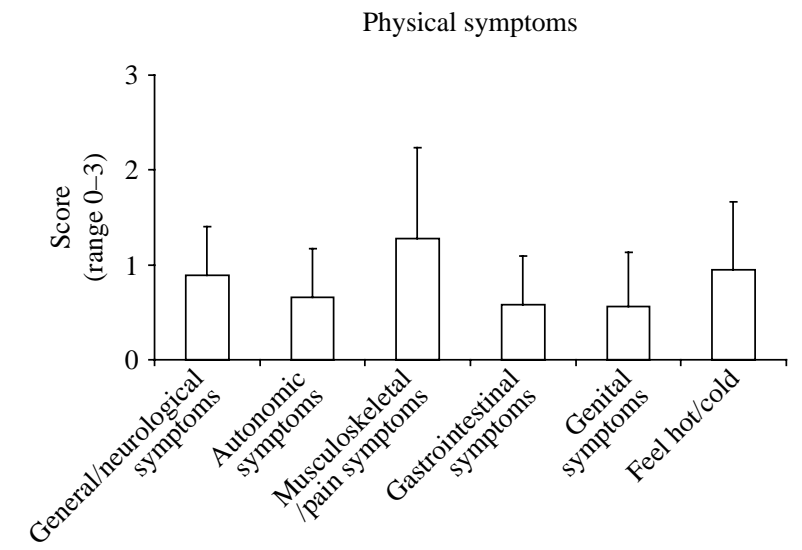

Figure 1 Scores of patients after long-term remission of Cushing's syndrome on the various organ systems measured by the physical symptom checklist. 
Table 2 Symptoms related to CS. Data are $n(\%)$, from the dimension 'illness identity' of the IPQ-R.

\begin{tabular}{lr}
\hline Symptoms & CS, $n=52$ \\
\hline Less muscle strength & $35(67 \%)$ \\
Less energy & $30(58 \%)$ \\
Gaining weight & $28(54 \%)$ \\
Concentration problems & $28(54 \%)$ \\
Vulnerable skin & $27(52 \%)$ \\
Memory impairment & $27(52 \%)$ \\
Sore joints & $25(48 \%)$ \\
Bad physical condition & $22(42 \%)$ \\
Muscle pain & $19(37 \%)$ \\
Slow wound healing & $19(37 \%)$ \\
Visual impairment & $13(25 \%)$ \\
Hair growth & $13(25 \%)$ \\
Hair loss & $9(17 \%)$ \\
\hline
\end{tabular}

\section{Illness perceptions in CS as measured with the IPQ-R}

Illness identity dimension More than half of the patients reported to suffer from symptoms such as gaining weight, less muscle strength, less energy, concentration problems, memory impairment, and vulnerable skin and believed that these symptoms are solely caused by CS (Table 2).

Causal attributions dimension In the last part of the questionnaire, patients after remission of CS reported relatively frequently psychological attributions as their perceived cause of CS. Risk factors and behavioral attributions were infrequently mentioned as being the cause of their illness.

Illness perception dimensions There were no differences in the IPQ-R dimensions between patients who had been treated by different treatment modalities (i.e. transsphenoidal surgery versus both transsphenoidal surgery and adrenalectomy versus adrenalectomy, and secondly additional radiotherapy versus no additional radiotherapy).

When using a linear regression model, gender and age were not associated with any of the illness perception dimensions. Duration of remission was also not associated with any of the illness perception dimensions, except for illness coherence, which constitutes the personal understanding of the disease $(\beta=-2.698, P=0.04)$. Furthermore, there were differences between patients with hydrocortisone substitution $(n=29)$ and patients without cortisol substitution $(n=23)$ in the perceived chronicity $(P=0.005)$ and fluctuations in the disease $(P=0.002)$. The clinical characteristics of patients with and without hydrocortisone substitution are detailed in Table 1.

In addition, hypopituitarism was associated with the number of symptoms attributed to the disease $(\beta=0.303$, $P=0.030)$, chronicity $(\beta=0.468, P=0.001)$, and fluctuations $(\beta=0.333, P=0.016)$ of the disease, and the perceived consequences $(\beta=0.323, P=0.019)$.

\section{Relationship between illness perceptions and QoL}

The scores of patients with CS on the various QoL questionnaires are depicted in Table 3. Levels of association between the IPQ-R dimensions and the QoL scales are shown in Table 4 as partial correlations, controlled for duration of remission.

The IPQ-R dimension illness identity showed positive correlations with the physical symptom checklist (PSC), mobility, activity, and anxiety, and inverse correlation with the VAS and the CushingQoL, all reflecting worse QoL when more symptoms are attributed to the disease on the illness identity dimension. The dimension timeline (acute/chronic) also showed an inverse correlation with the VAS and the CushingQoL. This indicates that patients who perceive their disease as chronic have a lower QoL. The dimension negative consequences showed a positive correlation with activity, and a negative correlation with the VAS and the CushingQoL. Furthermore, the emotional representations dimension showed a positive correlation with the PSC, mobility, and anxiety and an inverse correlation with the CushingQoL. The dimension personal control showed an inverse correlation with self-care and a positive correlation with the VAS. Perceived treatment control showed an inverse correlation with mobility and pain, and a positive correlation with the VAS and the CushingQoL. The dimension illness coherence (understanding of the disease) also showed an inverse correlation with mobility. Lastly, psychological attributions as a cause of CS showed a positive correlation with the PSC and mobility, but an inverse correlation with the CushingQoL. The dimensions timeline (cyclical), risk factors, and behavioral attributions did not correlate with any of the QoL questionnaires.

In addition, patients with low and high scores on the various QoL questionnaires were compared. The median value was used to define low versus high scores. Patients with high scores on the PSC (indicating a more

Table 3 QoL in patients with CS. Data are mean (S.D.). A higher score on the physical symptom checklist, mobility, self-care, activity, pain, and anxiety indicates a worse QoL, whereas higher scores on the visual analog scale (VAS) and the CushingQoL indicate a better QoL.

\begin{tabular}{lc}
\hline & CS, $n=49$ \\
\hline $\begin{array}{l}\text { Physical symptoms checklist } \\
\text { Total score }\end{array}$ & $40.8(26)$ \\
EQ-5D & \\
Mobility & $1.4(1)$ \\
Self-care & $1.1(0)$ \\
Activity & $1.7(1)$ \\
Pain & $1.8(1)$ \\
Anxiety & $1.4(1)$ \\
VAS & $65.5(18)$ \\
Cushing QoL & \\
Total score & $52(18)$ \\
\hline
\end{tabular}


Table 4 Partial correlations between the IPQ-R dimensions and QoL scales, controlling for duration of remission.

\begin{tabular}{|c|c|c|c|c|c|c|c|c|}
\hline & $\begin{array}{l}\text { Physical symp- } \\
\text { tom checklist }\end{array}$ & $\begin{array}{l}\text { EQ-5D } \\
\text { mobility }\end{array}$ & $\begin{array}{l}\text { EQ-5D } \\
\text { self-care }\end{array}$ & $\begin{array}{l}\text { EQ-5D } \\
\text { activity }\end{array}$ & $\begin{array}{l}\text { EQ-5D } \\
\text { pain }\end{array}$ & $\begin{array}{l}\text { EQ-5D } \\
\text { anxiety }\end{array}$ & $\begin{array}{l}\text { EQ-5D } \\
\text { VAS }\end{array}$ & $\begin{array}{c}\text { Cushing } \\
\text { QoL }\end{array}$ \\
\hline $\begin{array}{l}\text { Illness identity } \\
\text { Timeline (acute/chronic) } \\
\text { Timeline (cyclical) }\end{array}$ & $0.625^{\dagger}$ & $0.327^{*}$ & & $0.329^{*}$ & & $0.319^{*}$ & $\begin{array}{l}-0.382^{*} \\
-0.326^{*}\end{array}$ & $\begin{array}{l}-0.659^{\dagger} \\
-0.339^{\star}\end{array}$ \\
\hline Consequences & $0.413^{\dagger}$ & $0.313^{*}$ & & $0.317^{\star}$ & & $0.591^{\dagger}$ & $-0.411^{\dagger}$ & $\begin{array}{l}-0.316^{\star} \\
-0.629^{\dagger}\end{array}$ \\
\hline $\begin{array}{l}\text { Personal control } \\
\text { Treatment control } \\
\text { Illness coherence }\end{array}$ & 列 & $\begin{array}{l}-0.348^{*} \\
-0.353^{*}\end{array}$ & $-0.348^{\star}$ & & $-0.412^{\dagger}$ & & $\begin{array}{l}0.347^{*} \\
0.486^{\dagger}\end{array}$ & $0.326^{*}$ \\
\hline $\begin{array}{l}\text { Psychological attributions } \\
\text { Risk factors } \\
\text { Behavioral attributions }\end{array}$ & $0.412^{\dagger}$ & $0.336^{\star}$ & & & & & & $-0.327^{\star}$ \\
\hline
\end{tabular}

Only correlations that reached statistical significance $(P<0.05)$ are depicted, ${ }^{*} P<0.05$ and ${ }^{\dagger} P<0.01$.

impaired QoL, $n=25)$ attributed significantly more symptoms to CS $(P=0.002)$ than patients with a lower score $(n=24)$.

On the VAS, patients with a lower score (indicating a more impaired QoL, $n=25$ ) attributed more symptoms to $\operatorname{CS}(P=0.010)$ than patients with a higher score $(n=24)$. Patients with a lower score on the CushingQoL (indicating a more impaired QoL, $n=23$ ) attributed more symptoms to CS $(P<0.001)$, scored higher on timeline acute/chronic $(P=0.004)$, higher on consequences $(P<0.001)$, higher on treatment control $(P=0.010)$, and lower on emotional representations $(P<0.001)$. These analyses indicate that patients with a worse QoL report more affected illness perceptions than patients with a better QoL.

There were no significant differences in QoL between patients with and without hydrocortisone substitution (data not shown).

The above mentioned findings make clinical sense, in that the IPQ dimensions and the closely related QoL subscales measure the same concepts or concepts that logically interact with each other, i.e. diseaserelated symptoms and daily activities, or (negative) consequences and perceived well-being.

\section{Illness perceptions in CS compared with reference groups}

Illness perceptions in patients after long-term cure of CS compared with acute and chronic pain patients Illness perceptions of patients with CS were compared with acute pain patients. Patients with CS reported more illness-related complaints $(P=0.008)$, more chronicity and fluctuations in the disease (both $P<0.0001)$, perceived more negative consequences of the disease, and less personal $(P<0.0001)$ and treatment $(P=0.002)$ control. However, patients with CS had a better personal understanding of the disease $(P<0.0001)$ than patients with acute pain (Table 5 and Fig. 2).
In comparison with chronic pain patients, patients with CS reported less illness-related complaints $(P=0.003)$, perceived less negative consequences of the disease $(P<0.001)$, and reported a smaller likelihood to seek medical care $(P<0.0001)$. Patients with CS reported more treatment control $(P=0.002)$ and had a better personal understanding of the disease $(P<0.0001)$ than patients with chronic pain.

\section{Illness perceptions in patients after long-term cure} of CS compared with patients with a chronic disease Illness perceptions of patients with CS were also compared with patients with another chronic disease, i.e. COPD. Patients with CS reported less illness-related complaints $(P<0.001)$, less chronicity in the disease $(P=0.002)$, and perceived more treatment controllability $(P=0.001)$. However, patient's with CS perceived less personal controllability of the disease $(P<0.0001)$ than COPD patients (Table 5).

Illness perceptions in patients after long-term cure
of CS compared with vestibular schwannoma Illness perceptions of patients with CS were compared with patients suffering from untreated vestibular schwannoma. Patients with CS reported more illness-related complaints $(P=0.002)$ and more chronicity in the disease $(P=0.004)$. Furthermore, patients with CS reported more negative consequences of the disease $(P<0.0001)$ and perceived less personal control of the disease $(P=0.010)$ than patients with vestibular schwannoma (Table 5).

\section{Discussion}

This study was performed to assess the illness perceptions of patients after long-term remission of CS in relation to QoL parameters. This is the first time that this is addressed in endocrine diseases. The results indicate that affected illness perceptions and reduced QoL parameters are strongly related. Moreover, patients 
Table 5 Comparison of CS patients' IPQ-R scores to other populations. Data are presented as mean (S.D.).

\begin{tabular}{|c|c|c|c|c|c|}
\hline IPQ-R & $\begin{array}{c}\text { CS } \\
n=52\end{array}$ & $\begin{array}{c}\text { Acute } \\
\text { pain } \\
n=35\end{array}$ & $\begin{array}{c}\text { Chronic } \\
\text { pain } \\
n=63\end{array}$ & $\begin{array}{l}\text { COPD } \\
n=171\end{array}$ & $\begin{array}{c}\text { VS } \\
n=80\end{array}$ \\
\hline Illness identity & $3.8(3)$ & $2.8(2)^{\star}$ & $6.2(3)^{\star}$ & $5.6(3)^{\dagger}$ & $2.2(2)^{*}$ \\
\hline Timeline (acute/chronic) & $23.6(7)$ & $13.4(5)^{\dagger}$ & $23.1(4)$ & $26.7(4)^{\star}$ & $20.6(4)^{*}$ \\
\hline Timeline (cyclical) & $11.9(4)$ & $9.4(3)^{\dagger}$ & $12.9(4)$ & $12.1(5)$ & $10.6(4)$ \\
\hline Consequences & $20.4(5)$ & $14.2(4)^{\dagger}$ & $23.5(4)^{\dagger}$ & $19.3(6)$ & $16.4(2)^{\dagger}$ \\
\hline Emotional representations & $15.3(5)$ & $16.1(4)$ & $19.8(4)^{\dagger}$ & $14.1(7)$ & $15.3(4)$ \\
\hline Personal control & $16.9(6)$ & $22.9(4)^{\dagger}$ & $18.4(4)$ & $22.4(6)^{\dagger}$ & $19.1(3)^{\star}$ \\
\hline Treatment control & $16.7(5)$ & $19.4(3)^{*}$ & $14.2(3)^{\star}$ & $14.3(4)^{\star}$ & $16.9(3)$ \\
\hline Illness coherence & $17.0(3)$ & $9.3(3)^{\dagger}$ & $13.4(5)^{\dagger}$ & NA & $18.1(4)$ \\
\hline $\begin{array}{l}\text { Psychological attributions } \\
\text { (score range 9-45) }\end{array}$ & $17.9(8)$ & NA & NA & NA & NA \\
\hline Risk factors (score range 5-25) & $8.3(4)$ & NA & NA & NA & NA \\
\hline $\begin{array}{l}\text { Behavioral attributions } \\
\text { (score range } 2-10 \text { ) }\end{array}$ & $2.9(1)$ & NA & NA & NA & NA \\
\hline
\end{tabular}

after long-term remission of CS report more negative illness perceptions compared with several reference groups.

This explorative study demonstrates that patients in long-term remission after treatment for CS attribute more symptoms to (the aftermath of) their disease than patients with acute pain or vestibular schwannoma, but less than patients with chronic pain or COPD. Furthermore, patients with long-term cure of CS show more strongly held beliefs regarding the chronic nature of the condition compared with patients with acute pain or vestibular schwannoma, but less than COPD patients. Patients with long-term cure of CS also believe that their illness is more cyclical than patients with acute pain. Patients with long-term remission of CS report more negative consequences of the disease compared with patients with acute pain or vestibular schwannoma, but less negative consequences than patients with chronic pain. In addition, patients with long-term remission of CS are less likely to seek medical care than patients with chronic pain. Moreover, patients with long-term cure of CS have a lower perceived personal controllability of the disease compared with patients with acute pain, COPD, or vestibular schwannoma. Furthermore, patients with long-term cure of CS have a lower perceived treatment controllability of the disease than patients with acute pain, but a higher perceived treatment controllability than patients with chronic pain or COPD. Finally, patients with long-term remission of CS have a better personal understanding of their disease compared with patients with acute or chronic pain.

Patients' perceptions can be based on information from different sources. Therefore, patients' illness perceptions do not necessarily represent the medical status of the disease. This could explain why patients in remission of CS perceive their illness as chronic or cyclical and believe that a psychological attribution might have caused CS.
Patients with hydrocortisone dependency had stronger beliefs regarding the chronic cyclical nature of CS than patients without hydrocortisone dependency. Furthermore, hypopituitarism was associated with the number of symptoms attributed to CS, chronicity and fluctuations of the disease, and the perceived consequences of CS. Therefore, hydrocortisone dependency and hypopituitarism both influence illness perceptions.

This study also demonstrates that there is a strong relationship between illness perceptions and QoL. Affected illness perceptions are correlated with a more impaired QoL. This has already been observed in multiple other medical conditions (24). This relationship is a relevant observation, since patients with longterm cure of CS have persistent complaints reflected in impaired QoL (3-5). These complaints are often misunderstood and difficult to treat. Therefore, awareness of how these patients perceive their disease and its

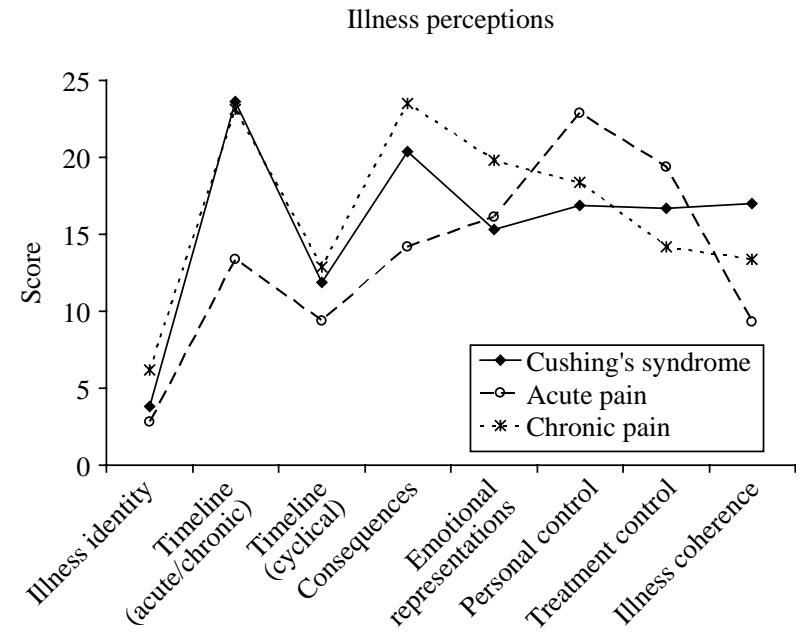

Figure 2 Scores on the IPQ-R of patients after long-term remission of CS, patients with acute or chronic pain. 
consequences could lead to better understanding of CS and its long-term effects. Furthermore, the current data on persisting perceptions of a chronic, rather than of a cured disease in combination with altered coping strategies in these patients (6) permit the design of an intervention based on combinations of strategies including cognitive behavioral therapy, self-management training, and information on the negative effects of the disease. Self-management training involves exploring, eliciting, and changing illness perceptions of the patient, which in turn determines coping styles and self-management behavior $(7,25)$. The intervention should be led by a health psychologist and endocrinologist and includes topics such as information about the illness and treatment, beliefs about consequences, beliefs about personal control, beliefs in selfefficacy, and the role of the social network (26). We speculate that with a targeted intervention, patients could be taught self-management skills and be better informed about the consequences of their disease. We believe that this approach might lead to an improved QoL, since this study shows that illness perceptions and QoL are strongly correlated. Similar self-management and educational interventions are currently available for patients with, e.g. inflammatory bowel disease (27) and after stroke (28). Increasingly, partners of patients are involved in such self-management training, with encouraging results; the partners help in ensuring that the patient actually performs self-management skills in the home situation (26).

A possible limitation of this study might be the fact that the symptoms commonly occurring in CS, which are described in the illness identity dimension, were not validated but instead based on the input of experienced endocrinologists. However, these symptoms were not used in the comparison of patients with CS versus the patients from the reference samples and, therefore, do not influence the results of this study. A second limitation is the possibility that the difference in age distribution between the various groups might have affected the results. Nonetheless, in this study, age did not have an effect on illness perceptions. However, this does not necessarily control for the possibility of age as a confounder because of the relatively small sample size. Another possible limitation of this study is the use of convenience reference samples. Illness perceptions have never been studied in patients with endocrine diseases before, and therefore, it was not straightforward to compare our patients to patients with other endocrine diseases. In the current explorative study, we decided to invite a large sample of patients with CS and to compare them with existing available reference samples. Future studies examining the differences in illness perceptions between patients with various (endocrine) disorders in larger samples should include correction for possible confounders such as age and gender.

In summary, there is a strong correlation between illness perceptions and decreased QoL. Patients after long-term remission of CS reported more negative illness perceptions compared with various reference samples. These results strongly point toward the need to develop, to apply and to evaluate a self-management and/or educational intervention aimed to improve these illness perceptions and thereby QoL in patients after remission of CS.

\section{Declaration of interest}

The authors declare that there is no conflict of interest that could be perceived as prejudicing the impartiality of the research reported.

\section{Funding}

This research did not receive any specific grant from any funding agency in the public, commercial, or not-for-profit sector.

\section{References}

1 Brown ES. Effects of glucocorticoids on mood, memory, and the hippocampus. Treatment and preventive therapy. Annals of the New York Academy of Sciences 20091179 41-55. (doi:10.1111/j. 1749-6632.2009.04981.x)

2 Fietta P, Fietta P \& Delsante G. Central nervous system effects of natural and synthetic glucocorticoids. Psychiatry and Clinical Neurosciences 200963 613-622. (doi:10.1111/j.1440-1819. 2009.02005.x)

3 Tiemensma J, Kokshoorn NE, Biermasz NR, Keijser BJ, Wassenaar MJ, Middelkoop HA, Pereira AM \& Romijn JA. Subtle cognitive impairments in patients with long-term cure of Cushing's disease. Journal of Clinical Endocrinology and Metabolism 201095 2699-2714. (doi:10.1210/jc.2009-2032)

4 Tiemensma J, Biermasz NR, Middelkoop HAM, van der Mast RC, Romijn JA \& Pereira AM. Increased prevalence of psychopathology and maladaptive personality traits after long-term cure of Cushing's disease. Journal of Clinical Endocrinology and Metabolism 201095 E129-E141. (doi:10.1210/jc.2010-0512)

5 van Aken MO, Pereira AM, Biermasz NR, van Thiel SW, Hoftijzer HC, Smit JW, Roelfsema F, Lamberts SW \& Romijn JA. Quality of life in patients after long-term biochemical cure of Cushing's disease. Journal of Clinical Endocrinology and Metabolism 200590 3279-3286. (doi:10.1210/jc.2004-1375)

6 Tiemensma J, Kaptein AA, Pereira AM, Smit JWA, Romijn JA \& Biermasz NR. Coping strategies in patients after treatment for functioning or non-functioning pituitary adenomas. Journal of Clinical Endocrinology and Metabolism 201196 964-971. (doi:10. $1210 /$ jc. 2010-2490)

7 McAndrew LM, Musumeci-Szabo TJ, Mora PA, Vileikyte L, Burns E, Halm EA, Leventhal EA \& Leventhal H. Using the common sense model to design interventions for the prevention and management of chronic illness threats: from description to process. British Journal of Health Psychology 200813 195-204. (doi:10.1348/ 135910708X295604)

8 Leventhal H, Meyer D \& Nerenz D. The common sense representation of illness danger. In Contributions to Medical Psychology, pp 17-30. Ed. S Rachman, New York: Pergamon Press, 1980.

9 Petrie KJ, Cameron LD, Ellis CJ, Buick D \& Weinman J. Changing illness perceptions after myocardial infarction: an early intervention randomized controlled trial. Psychosomatic Medicine 200264 $580-586$.

10 Pereira AM, van Aken MO, van Dulken H, Schutte PJ, Biermasz NR, Smit JW, Roelfsema F \& Romijn JA. Long-term predictive value of postsurgical cortisol concentrations for cure 
and risk of recurrence in Cushing's disease. Journal of Clinica Endocrinology and Metabolism $2003 \mathbf{8 8}$ 5858-5864. (doi:10. 1210/jc.2003-030751)

11 Moss-Morris R, Weinman J, Petrie K, Horne R, Cameron L \& Buick D. The Revised Illness Perception Questionnaire (IPQ-R). Psychology and Health 200217 1-16. (doi:10.1080/08870440 290001494)

12 Weinman J, Petrie K, Sharpe N \& Walker S. Causal attributions in patients and spouses following a heart attack and subsequent lifestyle changes. British Journal of Health Psychology 20005 263-273. (doi:10.1348/135910700168900)

13 Fowler C \& Baas LS. Illness representations in patients with chronic kidney disease on maintenance hemodialysis. Nephrology Nursing Journal 200633 173-186.

14 Fischer M, Scharloo M, Abbink J, van 't Hul A, van Ranst D, Rudolphus A, Weinman J, Rabe K \& Kaptein AA. The dynamics of illness perceptions: testing assumptions of Leventhal's commonsense model in a pulmonary rehabilitation setting. British Journal of Health Psychology 201015 887-903. (doi:10.1348/ 135910710X492693)

15 Hirsch D, Ginat M, Levy S, Benbassat C, Weinstein R, Tsvetov G, Singer J, Shraga-Slutzky I, Grozinski-Glasberg S, Mansiterski Y, Shimon I \& Reicher-Atir R. Illness perception in patients with differentiated epithelial cell thyroid cancer. Thyroid 2009 19 459-465. (doi:10.1089/thy.2008.0360)

16 Callaghan B, Condie E \& Johnston M. Using the common sense self-regulation model to determine psychological predictors of prosthetic use and activity limitations in lower limb amputees. Prosthetics and Orthotics International 200832 324-336. (doi:10. 1080/03093640802242326)

17 Searle A, Norman P, Thompson R \& Vedhara K. A prospective examination of illness beliefs and coping in patients with type 2 diabetes. British Journal of Health Psychology 200712 621-638. (doi:10.1348/135910706X164935)

18 American Psychiatric Association. In Diagnostic and Statistical Manual of Mental Disorders, 3rd edn. Washington D.C.: APA, 1980.

19 de Waal MW, Arnold IA, Spinhoven P, Eekhof JA \& van Hemert AM. The reporting of specific physical symptoms for mental distress in general practice. Journal of Psychosomatic Research 200559 89-95. (doi:10.1016/j.jpsychores.2005.02. 011)
20 The EuroQol Group. EuroQol - a new facility for the measurement of health-related quality of life. Health Policy 199016 199-208. (doi:10.1016/0168-8510(90)90421-9)

21 Webb SM, Badia X, Barahona MJ, Colao A, Strasburger CJ, Tabarin A, van Aken MO, Pivonello R, Stalla G, Lamberts SW \& Glusman JE. Evaluation of health-related quality of life in patients with Cushing's syndrome with a new questionnaire. European Journal of Endocrinology 2008158 623-630. (doi:10.1530/EJE07-0762)

22 Vogel JJ, Godefroy WP, van der Mey AG, Le Cessie S \& Kaptein AA. Illness perceptions, coping, and quality of life in vestibular schwannoma patients at diagnosis. Otology $\mathcal{E}$ Neurotology 2008 29 839-845. (doi:10.1097/MAO.0b013e3181820246)

23 Scharloo M, Kaptein AA, Schlosser M, Pouwels H, Bel EH, Rabe KF \& Wouters EF. Illness perceptions and quality of life in patients with chronic obstructive pulmonary disease. Journal of Asthma 200744 575-581. (doi:10.1080/02770900701537438)

24 Petrie KJ, Jago LA \& Devcich DA. The role of illness perceptions in patients with medical conditions. Current Opinion in Psychiatry 200720 163-167. (doi:10.1097/YCO.0b013e328014a871)

25 Kaptein AA, Klok T, Moss-Morris R \& Brand PL. Illness perceptions: impact on self-management and control in asthma. Current Opinion in Allergy and Clinical Immunology $2010 \mathbf{1 0}$ 194-199. (doi:10.1097/ACI.0b013e32833950c1)

26 Jansen DL, Heijmans M, Rijken M \& Kaptein AA. The development of and first experiences with a behavioural self-regulation intervention for end-stage renal disease patients and their partners. Journal of Health Psychology $2011 \quad 16$ 274-283. (doi:10.1177/1359105310372976)

27 Barlow C, Cooke D, Mulligan K, Beck E \& Newman S. A critical review of self-management and educational interventions in inflammatory bowel disease. Gastroenterology Nursing 201033 11-18. (doi:10.1097/SGA.0b013e3181ca03cc)

28 Jones F \& Riazi A. Self-efficacy and self-management after stroke: a systematic review. Disability and Rehabilitation 201133 797-810. (doi:10.3109/09638288.2010.511415)

Received 7 April 2011

Revised version received 11 July 2011

Accepted 28 July 2011 